\title{
Linx
}

Revue des linguistes de l'université Paris X Nanterre

$41 \mid 1999$

L'hypothétique

\section{L'hyperterminotique, un modèle paradigmatique d'organisation du savoir}

Jean-Nicolas de Surmont

\section{(2) OpenEdition}

Journals

Édition électronique

URL : http://journals.openedition.org/linx/808

DOI : $10.4000 / \operatorname{linx} .808$

ISSN : 2118-9692

Éditeur

Presses universitaires de Paris Nanterre

\section{Édition imprimée}

Date de publication : 1 décembre 1999

Pagination : 139-161

ISBN : 0246-8743

ISSN : 0246-8743

\section{Référence électronique}

Jean-Nicolas de Surmont, «L'hyperterminotique, un modèle paradigmatique d'organisation du savoir », Linx [En ligne], 41 | 1999, mis en ligne le 27 août 2012, consulté le 19 avril 2019. URL : http:// journals.openedition.org/linx/808; DOI : 10.4000/linx.808

Ce document a été généré automatiquement le 19 avril 2019

Département de Sciences du langage, Université Paris Ouest 


\title{
L'hyperterminotique, un modèle paradigmatique d'organisation $\mathrm{du}$ savoir
}

\author{
Jean-Nicolas de Surmont
}

1 C'est à la philosophie classique (l'arbre de Porphyre) que la théorie générale de la terminologie (dorénavant TGT) a emprunté la métaphore de l'arbre. En effet, l'arbre de domaine en terminologie essaie d'assigner une valeur monoréférentielle au terme. Cette préoccupation méthodologique est l'un des principes directeurs de la TGT élaborée par l'autrichien Eugen Wüster. Depuis Eugen Wüster, l'idéal terminologique a été de donner une dénomination à un concept (biunivocité du terme). Selon cet aspect de la théorie terminologique, la spécificité d'une unité terminologique repose sur la relation privilégiée qu'elle entretient avec d'autres unités d'un domaine de savoir et sur le fait qu'elle sert par là-même à établir un modèle standardisé de représentation du savoir.

Ce « volet » de la TGT constitue une problématique dominante en intelligence artificielle : les systèmes de représentation des savoirs. Dans cette contribution, nous tenterons de démontrer que la représentation linéaire de la connaissance ne peut pas rendre compte de la nature polyèdre du terme. Cela nous conduira à parler de ce que j'ai baptisé l' hyperterminotique et à donner une vision générale de quelques courants ayant cours en métaterminologie, comme l'intérêt croissant pour les aspects sociologiques et épistémologiques du terme. Nous traiterons par le fait même d'aspects philosophiques du traitement des terminologies à travers le réseau internet. A l'intérieur de la métaterminologie, l'hyperterminotique annonce des développements imminents dans l'acquisition de la terminologie.

\section{Pourquoi créer la dénomination hyperterminotique?}

Le terme hyperterminotique est construit à partird'hyperterminologie, emprunt que je fais à Budin et al. (1994) et aussi de terminotique (1977), employé, pour la première fois, selon l'information disponible, par Brian Harris ${ }^{1}$ et plus tard par P. Auger et D. Goaudec. 
Terminotique comporte lui-même la notion d'informatique et le suffixe -tique spécifique, selon Caroline de Schaetzen, aux disciplines automatisées. Celle-ci souligne en effet que l'informatique, en touchant un secteur d'activité et de connaissance, crée de nouvelles disciplines multidisciplinaires. Les signes de l'importance du statut disciplinaire de ces nouvelles recherches ont nécessité, poursuit-elle, l'ajout du suffixe -tique à la langue française : «Elle a pratiqué une aphérèse sur le mot automatique: tronqué au départ de son extrémité droite, ce mot est désormais compris comme un suffixe signifiant 'informatisation de et science de l'informatisation de' la maison (domotique), la documentation (documentique), la traduction (traductique), des activités de bureau ( bureautique) $\aleph^{2}$. Elle ajoute que l'emprunt à l'anglais de ce suffixe ne serait attesté que dans bureautics. C'est donc à la lumière de ces considérations sur l'instruction sémantique que nous comprenons mieux la création du terme hyperterminotique qui concerne donc les situations ou processus d'organisation et de gestion de termes et de terminologies par le biais d'outils hypertextuels à l'intérieur d'un réseau.

4 Jusqu'à maintenant l'ordinateur s'est avéré le seul moyen de traiter des données terminologiques de manière hypertextuelle. Il s'avère donc impératif de recourir à la néologie dénominative parce que nul terme jusqu'à présent n’a désigné précisément l'activité d'organiser la terminologie par l'usage de l'informatique. L'usage nécessaire de l'ordinateur dans le traitement hypertextuel des unités terminologiques m'encourage à employer dans un cadre théorique le terme hyperterminotique, construit à partir de la combinaison des mots hypertexte ${ }^{3}$, terminologie et informatique plutôt que hyperterminology qui avait été jusqu'à présent utilisé dans des contextes similaires. L'hyperterminology (que nous traduisons librement par hyperterminologie) devrait en fait désigner les procédés hypertextuels comme les corélats, les renvois analogiques, etc. Enfin, le besoin de création d'un nouveau concept repose non tant sur l'existence ou non d'unités lexicales ayant des degrés divers de technicité que sur les manières nouvelles de les organiser et surtout de les diffuser. C'est le besoin de décrire des pratiques terminologiques nouvelles qui nécessite la création du concept hyperterminotique : d'une part il s'agit de théoriser les usages, maintenant fréquents, des liens hypertextes, d'autre part, d'expliquer les tentatives concrètes, entre autres par l'équipe de Teresa Cabré et d'Arcadio Rojo (1996) à l'Institut Universitari de Lingüistica Aplicada (IULA) à Barcelone, d'organiser la terminologie sur le web à partir de principes métaterminologiques et des techniques de conception hypertextuelle, de communication télématique, qui conduisent donc à des pratiques terminologiques nouvelles mettant en avant des prises de décision.

\section{Considérations épistémologiques sur I'hyperterminotique}

\subsection{Ordinateur et métaterminologie}

5 Au sein du virage informatique en linguistique dans les années $1970^{4}$, la terminologie a brisé des frontières, spécialement avec l'émergence des banques de données terminologiques, qui ont crû de 14 en 1978 à 80 en 1994, montrant en même temps le rapide développement de la terminotique.

6 La pensée hypertextuelle systématisée par Vanevar Bush en 1945 et la métaterminologie élaborée dans les mêmes années partagent une croyance à propos de la science, à savoir que la connaissance se développe cumulativement et qu'elle doit être gérée de telle 
manière que le repérage de ses données s'en trouve facilité. La création de la pensée hypertextuelle a été fondée sur l'idée d'association et de liens. Dans l'hyperterminotique, une relation étroite existe avec les aspects heuristique et épistémologique impliqués dans l'acquisition de la terminologie. John Mc Carthy (1985: 23) fait la distinction entre les aspects épistémologique et heuristique de l'intelligence artificielle. Selon lui, le premier traite de quelle information doit être représentée alors que le deuxième, l'intelligence artificielle, se focalise sur la manière dont l'information doit être représentée. En me référant à cette distinction, je crois que les possibilités heuristiques (Abramson, 1995: 7) 醫 rendues réalisables par l'ingénierie du savoir donnent de nouvelles perspectives épistémologiques sur la nature polyèdre du terme.

\subsection{Regards sur des courants métaterminologiques}

7 Les développements cruciaux soulevés par les nouveaux courants en terminologie sont par le fait même en jeu dans les orientations du modernisme occidental (pensée fragmentaire, déconstruction, phénomène des quanta). Le traitement de la terminologie en transdisciplinarité s'inscrit dans une sociologie du savoir (Voir Robertson, 1996; Breton, 1992).

8 La socioterminologie, développée en France principalement à l'Université de Rouen, fait partie des récentes réflexions qui traduisent des changements épistémologiques dans la réflexion métaterminologique. A ce propos Louis Guespin (1990:645) montre une série d'oppositions entre la TGT et la socioterminologie :

- la concentration sur l'univocité versus la description de la variété des interactions scientifiques et techniques ;

- les wüstériens «s'appuyaient sur un comité de spécialistes [...] afin de fixer leur terminologie alors que d'une autre côté, dans l'approche socioterminologique nous devons négocier avec les emplois variés qui apparaissent dans le discours. $»^{5}$

- finalement que la méthode de la TGT classifie les termes à l'intérieur d'un système conceptuel, alors que la socioterminologie utilise l'analyse du discours dans différentes interactions qui impliquent de prendre en compte les pratiques locales de la science et de la technologie.

9 Les sciences collaborent et empruntent des termes provenant d'autres disciplines (créant ainsi des métaphores scientifiques) ${ }^{6}$. Les nouvelles idées émergent dans les études en sciences sociales, l'expansion des ouvrages de vulgarisation, considérés comme des manifestations de la modernité (voir Breton, 1992), apportent à la théorie terminologique de nouvelles perpectives promues par les socioterminologues, principalement en France, tel que F. Gaudin (1993: 255), dont les seuls aspects logiques ne sont pas capables de rendre compte. Comme le signale Myriam Bouveret, l'une des positions de la socioterminologie concerne le concept envisagé comme une construction de connaissances, ce qui semble coïncider avec l'idée de construction du savoir propre à l'exploration hypertextuelle. Les deux techniques ne conçoivent pas la construction sociale et interactive au même niveau ; l'une, la socioterminologie, permet une meilleure compréhension du phénomène de négociation sémantique, c'est-à-dire l'usage, la diffusion, le réglage de sens et la pérennité des sémèmes en terminologie, l'autre, l'hyperterminotique envisage strictement le rapport homme-machine dans son aspect anthropologique et herméneutique. 


\subsection{Théorie générale de la terminologie}

10 L'objectif de celui qui est reconnu comme le fondateur de la terminologie, Eugen Wüster, était très clair : promouvoir les sciences avec l'aide d'un langage unifié et unifiant. Il voulait offrir une communication non-ambiguë à travers l'enregistrement et la description de termes et la normalisation des terminologies. L'idée de rassembler les disciplines scientifiques dans un même schème (Voir Esteban, 1996) s'inscrivait à l'intérieur d'un mouvement plus large d'idées espérantistes, d'investigations sur le langage et la réalité (Felber, 1994: 41) et subissait l'influence du positivisme logique. Selon Wüster, la logique conceptuelle avait clarifié les relations entre les concepts, les ordonnant de telle sorte qu'une discipline apparaissait (Oeser, 1994:26). Cela répondait aussi aux nécessités sociales en normalisation technique du langage. La terminologie classique (sur laquelle est construite la TGT) est basée sur l'équivalence entre les concepts et les signes (univocité entre le signe et la dénotation ${ }^{7}$ ), la précision et la cohérence. Cet idéal positiviste issu de la logique était aussi près des idées contemporaines des théories de Sčerba en lexicographie bilingue. Rita Timmerman soutient : «L'étude scientifique du vocabulaire de spécialité est confondue avec l'activité pragmatique de la standardisation. L'urgence à vouloir standardiser est le résultat de l'objectivisme qui est profondément inscrit dans la tradition philosophique et culturelle de l'Occident. $»^{8}$

11 Dans l'approche onomasiologique, l'arbre hiérarchique est développé et identifié par les définitions terminologiques. Constituer un objet c'est le dériver d'autres concepts. Suivant les hypothèses de l'École de Vienne, Merten et l'École Belge de terminologie représentée par D. Blampain, M. Van Campehoudt (Merten, 1992) et autres, ont développé un projet de recherche appelé TERMISTI qui adopte un tel paradigme mais le relativise également en reconnaissant des variations sémantiques dans les unités d'une microdiscipline à une autre et dans différentes langues. Même s'ils ont opté pour un travail descriptif plutôt que celui prescriptif de Wüster, ils continuent d'édifier des réseaux notionnels hiérarchisés.

\subsection{Interprétation vs représentation/organisation}

12 Si nous admettons que la représentation du savoir a un sens philosophique (quel sens rattachons-nous aux concepts ou aux objets?) et psychologique (quelles relations, associations établissons-nous entre ces objets?) nous devons considérer l'hyperterminotique comme relevant, dans notre perspective, de considérations philosophiques, voire métaphysiques. D'un autre côté, l'hyperterminotique a la spécificité de n'établir aucune relation hiérarchique entre les nœuds. Pour cette raison nous écartons l'usage du concept opératoire représentation qui nous apparaît trop proche de la notion de "hiérarchisation » et de "modèle structuré ». Mais s'il ne s'agit pas d'une représentation des connaissances, comment peut-on alors dénommer les rapports du réseau réticulaire? On est en fait, avance François Rastier, face à un abus de langage «car les représentations informatiques ne sont en fait que des transcriptions partielles, dans d'autres codes sémiotiques, des échanges sémiotiques à l'œuvre dans les pratiques sociales où elles prennent place. ${ }^{9}$ Choisir le terme interprétation, alors, inclurait les deux sens antérieurs de représentation mais n'exprimerait pas l'idée d'ensemble de nœuds impliqué par l'existence d'un treillis. Il convient certes au régime cognitif qui caractérise la navigation paradigmatique. En revanche il ne convient pas à exprimer la notion 
d'« association de nœuds ». En utilisant le terme organisation, nous désirons en fait purger les traits sémantiques impliquant la notion de « hiérarchie » qu'expriment normalement représentation et structure en métaterminologie. Ce dernier terme est aussi fortement connoté par la notion de « système » dans son acception politique et épistémologique. La notion de « système » implique elle aussi celle d'organisation structurée et la philosophie lui rattache traditionnellement une connotation laudative. Pour toute ces raisons, c'est le syntagme organisation du savoir qui nous apparaît plus pertinent pour la description des relations analogiques entre des nœuds.

13 Cette préférence théorique et terminologique adopte par ailleurs comme hypothèse (Condamines, 1994: 32) l'idée que le référent est d'abord saisi par les perceptions et n'appartient donc pas de ce fait à un système, contrairement aux concepts. La faiblesse de Wüster était en effet de croire en l'équivalence entre le système conceptuel et le système linguistique et ce même si les représentations linguistiques ne sont pas équivalentes dans toutes les langues (Rastier, $1995: 40$ ).

Enfin, nous reprenons le point de vue de Lerat (1997: 11) selon lequel connaissance équivaut ici à information.

\section{Le paradigme de l'hyperterminotique}

15 En utilisant le paradigme de la nouvelle organisation hypermédia du savoir, développé récemment en Espagne ${ }^{10}$ par l'anthropologue Arcadio Rojo et la linguiste Maria Teresa Cabré, et sur lequel s'est basé notre réflexion de départ, une compréhension de ces avancées technologiques et épistémologiques est possible. La réflexion de Rojo sur l'hypertexte provient de ses recherches antérieures (voir 1992: 209ss), inspirées des travaux de Robert Cavalier, dans lesquelles il discute le conflit entre l'éthique dans le monde occidental et l'ingénierie d'invention du savoir. Cabré (voir ses précédents travaux sur la diversité de la terminologie, la terminologie étant entendue comme une unité terminologique et comme métaterminologie : $1993: 31,37,82 ; 1996: 18)$ dit avoir été influencé par le catalaniste Antoni M. Badia i Margarit dans sa façon de focaliser les objets d'études à partir de différentes perspectives. La prochaine section examine entre autres la notion de circular text de Rojo et tentera de mesurer la contribution théorique de Rojo et Cabré à la théorie de l'organisation de la terminologie.

\subsection{Linéarité vs circularité}

Plusieurs publications (Piotrowski, Rojo, entre autres) reliant la terminologie à des considérations épistémologiques ont mis en relief l'idée de non-linéarité et de "non séquentialité" de la lecture hypertextuelle. D'une certaine manière, dans cette perspective, l'hypertexte peut être considéré comme un texte n'ayant ni début et ni fin évoquant la doctrine de l'éternel retour. La littérature sur l'hypertexte a adopté ces figures de l'éternel retour et du cercle. En fait ce sont davantage les liens eux-mêmes qui construisent le réseau jusqu'à l'infini, constituant un espèce d'architexte, le texte demeurant toujours présenté de manière linéaire. Pour cette raison, Rojo et Cabré préfèrent l'appeler circular text (1996: 417). En adoptant ce point de vue, nous aurions cependant toujours à considérer le problème que pose l'ambiguité notée par Henri Meschonnic (1996: 19) et Levy (1995: 203) entre l'ouverture ou la fermeture du savoir encyclopédique. Nous aurions aussi à spécifier la différence entre la circularité linguistique 
d'un dictionnaire, qui est le résultat du dictionnaire comme un texte, ce qui signifie fermé sur sa propre nomenclature ${ }^{11}$, et la circularité cognitive qui semble davantage rendre compte d'un processus d'intelligibilité ouverte, comme nous le laisse entendre les travaux d'Umberto Eco (cf. Opera aperta). Ainsi la circularité signifie-t-elle la totalité ou plutôt une organisation infinie.

17 En nous référant à l'article de Jacob Neusner (1997 : 375 ss.) sur la pensée paradigmatique versus la pensée historique, nous ne devrions pas considérer la pensée hypertextuelle comme un mode circulaire de pensée mais plutôt comme la base d'un paradigme cognitif du traitement des unités terminologiques. Dans le cas de l'hyperterminologie nous pourrions ainsi parler d'organisation paradigmatique des unités terminologiques. J'expliquerai plus loin ce que j'entends par paradigme. Pour l'instant revenons à cette notion de circularité. Une exploration circulaire de n'importe quelle conception hypertextuelle impliquerait retourner vers l'arrière jusqu'au premier nœud d'information et alors statuer que l'exploration précédente apparaîtrait encore et encore ad infinitum et donc par le fait même qu'il n'y a pas de centre. Ce n'est pas tant la notion de circularité au sein de l'exploration hypertextuelle - rappelant le symbole de l'ouroboros , le serpent qui se mord la queue - qu'il convient évoquer, mais plutôt le fait que c'est l'internaute qui se situe lui-même au centre des choix à opérer sur la route à suivre. D'un autre côté, dé-linéariser ne veut pas automatiquement dire circularité, comme nous pouvons l'observer à la lecture de Budin et al. En effet, dans leur article sur l'hyperterminologie, le premier sur le sujet, ils utilisent dé-linéariser dans le sens de décontextualiser (1994:6), se référant à la relation entre le texte et le terme. Le texte est considéré comme étant linéaire alors que le terme, parce qu'il s'agit en quelque sorte du résultat d'une fragmentation du texte, est considéré comme dé-linéarisé. Dans cette perspective, l'hyperterminologie n'est pas abordée d'un point de vue métaphysique, épistémologique ou heuristique mais davantage en tenant compte de préceptes techniques portant sur les possibilités de traitement via une organisation télématique.

18 A cause de la nature paradigmatique du traitement de la terminologie à travers la navigation hypertextuelle, l'exploration peut être considérée comme un processus d' endoculturation (ou d'acculturation). La langue, et plus précisément le vocabulaire terminologique, apparaît comme condition de la culture (Lévy -Strauss : 1987) qui rétablit en fait, contrairement aux volontés standardisatrices de la terminologie classique, les critères de valeurs culturelles et de diversité des termes. La cyclicité est donc rejetée en faveur d'une organisation relationelle-réticulaire des termes entre eux à la manière d'un treillis. La métaphore de la navigation (qui est aussi sous-entendue dans Netscape navigator ), bien connue des internautes, nous rappelle, souligne Chatelain (1996: 169), celle de Francis Bacon, proficience, qui renvoie à une idée de mouvement et de marche vers le savoir, d'acquisition de compétences herméneutiques, plutôt qu'une expression statique de celui-ci, comme si la pensée construisait le savoir. Dans les termes du philosophe P. Lévy (1995 : 176), nous pourrions avoir accès à un espace de savoir plutôt qu'à des frontières épistémologiques bien délimitées qui façonnent des territoires. Nous renvoyons encore ici non seulement à l'accès multidimensionnel et multicritériel au savoir (le nœud est ancré via plusieurs liens) mais aussi paradoxalement, puisque les phénomènes sont contemporains, à la multiplication des disciplines.

La construction de l'hyperterminotique a donc trait à une synthèse dynamique (comme une cuvre ouverte) en perpétuel mouvement des activités sociales et scientifiques. L'hypertexte consiste alors «en une structure d'interaction récursive entre textes 
(documents) et interprétations "temporairement stabilisées" (ou connaissances) destinée à produire de nouvelles élaborations sémantiques (des interprétations) comme l'affirme David Piotrowski (1996 : 323).

\subsection{Hyperterminotique et complexité}

\subsubsection{Description de la réalité}

La TGT a développé une connaissance abstraite (systématicité, savoir cohérent) qui, jusqu'à maintenant n'a pas été en mesure de traiter des considérations empiriques comme la description complexe et la définition de la réalité ou du référent. Ahmad affirme à ce sujet: "Un vocabulaire scientifique [Kuhn] est constitutif du monde phénoménal dans le sens qu'il crée une taxinomie des genres correspondant aux concepts disponibles à une communauté de discours habitant dans le monde. ${\aleph^{12}}^{2}$. Louis Marin affirme quant à lui : " [...] la réalité, sinon la valeur objective, du signe, c'est son effacement par transparence devant l'idée et devant la chose signifiée ${ }^{13} \cdot{ }^{13}$ Ce n'est donc pas tant le signe réalité lui-même qui est indéfinissable ou encore la réflexion même sur le concept de réalité, en fait c'est plutôt l'accès au monde phénoménal sans représentation qui pose problème. Pour cette raison, les terminologues ne devraient pas utiliser le terme réalité, entendant par cela le monde en lui-même, épistémiquement inaccessible à l'apprenant. La théorie de la terminologie a aussi été très faible, pour parler des termes décrivant des réalités formelles. Si le référent est abstrait, que serait alors le concept, entendu qu'en conceptologie, le concept existe toujours indépendamment du terme. La perception de la réalité a été le point de départ des considérations sur l'univocité du terme.

\subsubsection{Savoir expert et savoir lexical : les différents degrés de scientificité}

21 L'hyperterminotique, concernée par la présentation de données terminologiques sur une page web, rejette la monoréférentialité du terme qui tend à isoler les champs du savoir, comme l'a développée l'École de Vienne. Cette nouvelle conception constitue une alternative à la TGT, non seulement en établissant différents réseaux notionnels comme l'ont adopté les membres de l'équipe de TERMISTI en Belgique et de Mayer, Geer et Henne (1990) ou en reliant différents tableaux (représentation graphiques bi-dimensionnelles) (Amer, 1994) qui demeurent des représentations hiérarchiques, mais aussi en mettant en relation différents points de vue sur le même domaine (relations polyhyérarchiques). En effet, certains termes qui appartiennent à un champ (comme l'économie politique) peuvent aussi être utilisé dans le domaine de l'énergie renouvelable. ${ }^{14}$

L'analyse des unités terminologiques nous montre qu'elles n'apparaissent pas uniquement dans un discours spécialisé. Les dictionnaires de langue générale ont ainsi souvent utilisé des textes littéraires afin d'illustrer des emplois et des sens scientifiques et techniques (voir Candel, 1990 : 47). Ainsi, une opposition absolue entre lexème et termes devient non valable. Depuis que nous savons que la spécificité du terme n'est pas invariable (Phal cité par Morgenroth, $1994: 13$ ), nous devrions aborder les termes d'une manière holistique (Handwerker (1989), Budin et al. 1994 : 3; Gentilhomme, 1994: 33) dans l'esprit d'une science de la complexité ${ }^{15}$ admettant la double fonction des termes: c'est-à-dire en distinguant le savoir lexical (unité-mot) et le savoir expert (unité-terme) (ou « vocabulaire d'orientation scientifique» (Morgenroth, 1994: 75), en prenant en compte 
les valeurs culturelles, le discours, les registres de langues. Chemin faisant, ces préceptes feraient transférer l'intérêt de la problématique du signe, corollaire à l'arborescence (unicité du chemin orienté du sommet à un nœud), à une problématique du texte, corollaire au treillis (pas de circuit orienté), et de la multidimensionalité. ${ }^{16}$ C'est dans cette optique que Lerot affirme : « Les langues naturelles organisent les concepts dans des hiérarchies emmêlées, appelées hétérarchies. $»^{17} \mathrm{~A}$ la lumière de ces données, nous devrions aussi, enfin, mieux définir ce que nous entendons par connaissance, information, etc.

\subsubsection{Point de vue anthropologique}

23 L'organisation hypertextuelle nous permet d'utiliser la navigation horizontale entre les nœuds d'information liant entre elles les pages web. L'anthropologue Arcadio Rojo, en empruntant les conceptions hindouistes et égyptiennes de l'histoire et de la réalité (l'unité dans la diversité - le thème majeur des Upanishads -, Diaz, 1982 : 17), modifie le critère traditionnel (relation verticale) utilisé afin de définir les relations entre les termes. Comme François Gaudin l'affirme, les sciences ne doivent pas être considérés comme des disciplines, mais comme un ensemble de nœuds (1993:252). Ces nouvelles méthodes heuristiques donnent une vision anthropologique de l'organisation des terminologies. Les termes ne sont plus uniquement vus comme servant à décrire un savoir homogène et standardisé mais plutôt sur le plan de la diversité des cultures (traditions), perspective par laquelle nous pouvons déceler des procédés d'endoculturation via la transmission orale et l'enseignement des termes. Ce point de vue situe dès lors notre problématique au sein de la dynamique modernité/tradition, comme le fait l'internationalisation de la culture via le web. Selon moi, l'accès aisé à la communication internationale, même si la qualité des sites web est inégale, nous permet de développer un savoir encyclopédique.

\subsection{Hyperterminotique : une définition éthique du concept de paradigme}

L'hyperterminotique est alors considérée comme partie intégrante d'un savoir d'invention et ce pour plusieurs raisons: premièrement, les termes doivent être considérées à l'intérieur d'une sémiotique textuelle, i.e. les limites culturelles, sociologiques et historiques, également les domaines dans lesquels ils apparaissent (Voir Candel, 1993, Rastier). En d'autres termes, prendre en compte ces aspects des termes comme s'ils étaient considérés comme de simples unités lexicales devrait aussi nous permettre de redéfinir notre propre définition de la culture ${ }^{18}$. De plus, les terminologies, les pages web et l'internaute sont ainsi perçus comme des concepteurs (science of design, Herbert Simon ${ }^{19}$ ) dans leur relation avec l'édition électronique. Dans cet aspect, la création terminologique (néonymie) et la transmission d'informations font partie d'un ensemble de décisions concernant les valeurs éthiques qui construisent le réseau.

Un terme a de la validité, comme un événement historique (Simmel, 1995 : 300) s'il est fixé dans une période, à l'intérieur d'une communauté d'interprétation, si avec d'autres nœuds, il forme une unité de compréhension selon le paradigme choisi par l'internaute et le concepteur. Construire le réseau (au contraire de l'arbre de domaine) ne donne pas priorité au spécialiste comme c'est le cas dans les principes généraux de la TGT mais est le résultat du travail combiné du terminologue, du spécialiste, du cogniticien et de 
l'internaute. Il est en effet le résultat d'un paradigme individuel de l'internaute et n'engage pas un consensus collectif, bien qu'il s'inscrive dans une culture d'interprétation et que le cogniticien (ou si l'on veut l'architecte de l'hypertexte), le terminologue et le spécialiste soient à l'origine de l'enregistrement des données terminologiques.

Si l'hypertexte effectue des relations entre les nœuds, c'est sur une base non-hiérarchique (Voir le concept de contenu nucléaire dans Eco, 1999) et sans vocation classificatoire; il permet un accès interne au savoir non-structuré des réseaux. A l'intérieur de l'hypertexte composé, chaque terme devient un nœud d'information constitutif d'un plus grand texte qui le transcende: "paradigms are at the same time pertinent without regard to consideration of scale and formulated without interest in matters of singular context $»^{20}$, comme c'est le cas de la narration séquentielle d'événements singuliers (pensée historique). Dans l'arbre de domaine, un ensemble de termes représente l'état de la terminologie dans un domaine et ainsi un paradigme (au sens de Kuhn), évidemment instable, de la représentation du savoir (Sager, 1990: 16, Otman, 1996: 127). Cela ne signifie pas automatiquement qu'un virage théorique (conceptuel) implique seulement un changement d'habitudes linguistiques (en particulier lexicales). Le terme paradigme dans cette vision est considéré comme l'état achevé d'un système terminologique et concret (Voir Ahmad, 1996:8) dans une certaine période de temps. Cette inscription dans la durée événementielle rend complexe et instable l'ensemble des hypothèses que constitue le paradigme ${ }^{21}$. Mais l'usage du terme paradigme semble inadéquat dans le domaine des sciences sociales, comme le souligne Gerhard Budin (1991: 338) : «L'interdépendance du changement conceptuel et de la modification des hypothèses, théories, etc. durant le processus de recherche est accompagné par un autre phénomène typique des sciences sociales et des humanités : au contraire des sciences naturelles telles que la physique, etc., ces disciplines n'ont pas de paradigmes (au sens de Kuhn) prédominants mais plutôt une pluralité de théories, hypothèses, école de pensée, etc. concernant le même 'objet'. Cela veut dire que plusieurs concepts différents sont formés à propos du même objet selon le cadre respectif de la théorie. $»^{22}$

C'est donc cette hétérogénéité de perspectives qui est proche de la nôtre et c'est en ce sens que nous employons le terme paradigme. Il n'a donc pas tellement une valeur universelle qu'il s'inscrit dans le cadre d'une perspective éthique et phénoménologique ${ }^{23}$, voire métaphysique statuant que naviguer au travers d'unités terminologiques à l'intérieur du web et créer des liens correspond à une série de prises de décisions éthiques et conceptuelles concernant le type d'information conceptuelle demandée à propos du terme. Sur le plan théorique, il rejoint le terme dans son acception linguistique dans la mesure qu'il met en relief différents segments qui peuvent figurer en un même point de la chaîne. ${ }^{24} \mathrm{~L}$ ' hétérogénéité des finalités des recherches montre donc qu'il y a en ce sens autant de paradigmes qu'il y a de manière d'appréhender les termes (stratégies de pensée) et d'abstraire le monde phénoménal, ce qui prouve en même temps que la terminologie est constitutive du monde phénoménal et non de la réalité, cette dernière étant inaccessible épistémiquement (Voir Borella, 1989 : 100).

La TGT a aussi basé ses hypothèses sur des critères hiérarchiques, en d'autres mots sur une problématique du signe provenant de la logique. Les développements actuels de la technologie, les nouvelles possibilités qu'offrent l'hypertexte dans le processus de requête d'information impliquent de reconsidérer les hypothèses et passer vers une problématique du texte selon la terminologie de Rastier (1996:12). La diffusion des mêmes unités terminologiques dans plusieurs disciplines, par le jeu de la métaphore par 
exemple mais aussi par l'usage d'une même unité dont le sens diffère selon le contexte d'utilisation représente un changement de point de vue selon Engel et Picht (1990:52) avec les traditionnelles banques de données et banques de documentation. Cette nouvelle façon de récupérer et de gérer des informations terminologiques consiste en une nouvelle orientation dans le processus d'organisation du savoir. ${ }^{25}$

Une conception rationaliste de l'action montre que le régime pragmatique de l'internaute est régi par des états mentaux, tels les croyances, désirs et intentions mais aussi par des opérations mentales telles que sélectionner, sauvegarder des informations en mémoire, comparer. Il voit donc, en définitive ce qu'il veut voir et choisit ce qu'il veut ou essaie de choisir, parfois malgré lui. Choisir une route de navigation serait en ces termes agir sur le monde phénoménal.

\section{Processus mentaux}

30 L'internaute peut établir presque toutes les relations ontologiques et logiques en terminologie: relation méronymique (partie-tout) (collection faite de), etc. mais sans distinction d'importance en ce qui a trait aux divisions hiérarchiques. Les liens peuvent conduire à d'autres liens, à des textes et donner au contenu nucléaire une information élargie (voir Eco 1999). ${ }^{26}$

31 S'inspirant des travaux de J. T. Guthrie, Tricot et al. affirment que la recherche d'information de manière hypertextuelle peut être modélisée ainsi : « formation d'un but cognitif, sélection d'une catégorie d'informations, extraction d'information, intégration à l'information préalablement extraite, recommencer jusqu'à ce que le but soit atteint. » $\mathrm{Ce}$ point de vue nous conduit vers la question des limites du sens du terme dans l'organisation du savoir renvoyant à l'idéal dictionnairique d'Abramson: "Un dictionnaire devrait être une banque de données encyclopédiques des mots d'une langue ou des langues couplées avec une méthode déductive d'accès et des méthodes de représentation multimédiatiques. $\aleph^{27}$ Dans ce sens, les textes dans lequel apparaît le terme, même s'ils sont considérés comme linéaires et conceptuels, appartiennent à un texte ouvert ou un ouvrage, et ne sont pas seulement des textes non linéaires liés entre eux comme dans les dictionnaires. Les termes bénéficieraient ainsi de nouveaux contextes. Cela n'est pas un objectif de résoudre les problèmes de définition des unités terminologiques. Les termes sont seulement montrés en contexte, comme cela est fait dans certains dictionnaires terminologiques. Nous commençons à voir le problème des niveaux de discours, qui renvoient, comme nous l'avons dit, au savoir lexical et au savoir expert. C'est un fait que les termes ne sont pas toujours utilisés dans des contextes scientifiques et techniques, dépendant de leur place dans un système conceptuel. Ainsi, un terme peut devenir relativement fréquent pour des raisons d'actualité (Morgenroth, 1994: 72), empruntant alors des caractéristiques du mot. Pourquoi parlons-nous donc encore de terminotique si en même temps nous remettons en question l'existence du contrôle de sens largement étudié par M. Bouveret (1996) dans sa thèse ? Dans la foulée de cette étude, c'est le regard multidisciplinaire sur les usages lexicaux qui ouvre le débat sur le statut des unités terminologiques. Cela serait certes le sujet d'un autre article qui apporterait les nuances nécessaires à la compréhension de ce problème complexe. La thèse de Myriam Bouveret apporte une contribution au comportement sémantique des unités lexicales et terminologiques. Néanmoins, nous pouvons affirmer que les mots de la langue générale n'apparaissent pas dans un sens technique alors que les termes, oui. C'est 
pour toutes ces raisons que nous n'avons proposé aucune distinction entre la sémantique linguistique et le savoir encyclopédique.

L'accès hyperterminotique et l'acquisition de la terminologie à travers le web sont vus comme un processus d'acculturation qui implique un ensemble de décisions éthiques prises par le concepteur et l'internaute. D'ultérieures études sur le sujet nous aideraient à comprendre si les arbres de domaine sont nécessaires pour comprendre les données terminologiques. ${ }^{28} \mathrm{Si}$ nous acceptons cette affirmation, nous devrions vérifier s'ils peuvent être le point de départ d'une compréhension holistique permise par l'organisationinterprétation telle que la télématique le permet. Si la métaterminologie s'est développée à l'intérieur d'une argumentation émanant de la logique, si l'intérêt pour la terminologie au XVIIIe et XIXe siècles est né des préoccupations des scientifiques face à la multiplication des dénominations - du fait des inventions et découvertes -, et de la nécessité de les systématiser, elle est maintenant influencée par la sémiotique culturelle, les considérations sociales, qui impliquent le problème de la diversité du discours et empruntent à d'autres sujets d'étude. Le paradigme scientifique dans le sens de Kuhn semble désormais inefficace à l'intérieur de l'interpénétration des sciences et des media, qui contribuent comme l'affirme Pierre Lévy «à enfler la sphère des signes sans attaches [territoriales]. $»^{29}$

33 A l'intérieur de cette communication, j'ai essayé de tracer un lien entre le savoir dit pur, l'objectivité, la représentation et une vue ontologique des termes qui deviennent à cet effet non plus représentés mais davantage, interprétés ou organisés. Cette recherche pourrait bénéficier des apports de la systémique, de la neurologie, mettant en relation les approches réticulaires et neuronales de l'organisation des cellules nerveuses avec les raisons qui nous portent à penser hypertextuellement.

\section{BIBLIOGRAPHIE}

ABRAMSON, H., (1995), « What is a hyperdictionary », TermNet News, $\mathrm{n}^{\circ} 49$, pp. 7-13.

AHMAD, K., (1996) « A terminology dynamic and the growth of knowledge : a case study in the nuclear physics and in the philosophy of science ", TKE '96, pp. 1-11.

AMER, A., (1984), « The effect of knowledge-map and underlining training on the reading comprehension of scientific texts ", English for Specific Purposes, vol. 13, n 1, pp. 35-45.

ASSY, Y., (1995), les Mots-clés de la linguistique dans les oeuvres de ses pionniers, thèse de doctorat d'état, Université de Nancy II, Villeneuve-D’Ascq, Presses Universitaires du Septentrion.

AUGER, P., (1989), « La terminotique et les industries de la langue », Meta, XXXIV, 3, pp. 450-456.

BERGENHOLT, H., (1995), « Dictionary structures » in Manual of Specialized Lexicography, the Preparation of Specialized Dictionaries, S. Tarp (ed.) Amsterdam/Phidadelphie, John Benjamins, pp. 188-223 (coll. Benjamins translation library, vol. 12).

BERK, E. and DEVLIN, J., (eds), (1991), Hypertext/Hypermedia Handbook, McGraw-Hill Publishing Company, New York, XVII. 
BORELLA, J., (1989), le Mystère du signe Histoire et théorie du symbole, Paris, Éditions de Maisonneuve et Larose, (coll. « Métalangage »).

BOUVERET, M., (1996), Néologie et terminologie : production de sens du terme, thèse de doctorat, Université Paul Valéry-Montpellier III,

BRETON, P., (1992), Utopie de la communication : l'émergence de l'homme sans intérieur, Paris, Éditions de la Découverte.

BUDIN, G., LUKAS, H. and STOCKINGER, J., (1994), « Ethnological knowledge and hypermedia : new ways of representing terminologies, concept and rituals ", Terminology, Science and Research, IITF-Journal, vol. $5 \mathrm{n}^{\circ} 2$, pp. 1-21.

BUDIN, G., (1991), « The application of terminology-based knowledge data bases in the humanities and the social sciences and its impact on research methods », in Computer in the Humanities and the Social Sciences Achievements of the 1980s, Prospects for the 1990s; Proceedings of the Cologne Computer Conference, Uses of the Computer in the Humanities and Social Sciences held at the University of Cologne, München, K. G. Saur, pp. 337-342.

CASSEYRE, J.-P., (1994), « Classements, classification et mode d'accès » in D. RENOULT (ed.), les Bibliothèques dans l'Université, Paris, Éditions du Cercle de la Librairie, pp. 167-176.

CABRE, M.-T., (1996), « Terminology today », in Terminology, LSP and Translation; Studies in Language Engineering in Honour of Juan C. Sager, Amsterdam/Phidadelphia, John Benjamins Publishing Company, pp.15-33.

CABRE, M.-T., (1993), La terminología, Teoría, metodología, aplicaciones, prólogo de J.-C. SAGER, Barcelona, Editorial Antartida/Empúries.

CANDEL, D., GERNER, H., BERNARDOFF, A. et BAUDINOT, P., (1990), « Aspect de la documentation scientifique et technique dans un grand dictionnaire de langue » in D. CANDEL, Dictionnairique et lexicographie, Autour d'un dictionnaire : le "Trésor de la langue française ", témoignages d'atelier et voies nouvelles, Paris, Didier Érudition, pp. 29-52.

CANDEL, D., (1993), « Français oral de spécialité et terminologie », Le langage et l'homme, vol. XXVIII, $n^{\circ} 4, n^{\circ}$ spécial « Socioterminologie », pp. 259-272.

CARR, M., (1997), « Internet dictionaries and lexicography », International Journal of Lexicography, vol. 10, n 3, Oxford University Press, pp. 209-230.

CHATELAIN, J.-M., (1996), « Du Parnasse à l'Amérique : l'imaginaire de l'encyclopédie à la Renaissance et à l'Âge classique », in R. SCHAER, pp. 156-163.

CONDAMINES, A., (1994), « Terminologie et représentation des connaissances », La Banque des mots, $\mathrm{n}^{\circ}$ spécial 6, pp. 29-44.

COOK, G., (1994), chapter 3 « A second bearing : AI theory and its limitations », in Discourse and Literature: The Interplay of Form and Mind, Oxford, Oxford University Press, pp. 59-94.

CZAP, H., and NEDOBITY, W., (ed.), (1990), TKE '90 : Terminology and Knowledge Engineering, Frankfurt, Indeks Verlag, 2 vol.

DE SAETZEN, C., (1997), « Chassé-croisé de vocables entre l'informatique et les discipines automatisées », in DE SAETZEN, pp. 123-135.

DE SAETZEN, C. (ed.), (1997), Terminologie et interdisciplinarité, Peeter, Louvain-la-Neuve.

DEWEY, M.,(1990), Abrégé de la classification décimale de Dewey ; nouvelle édition augmentée à partir de la première version intégrale française et de la XIXe édition intégrale en langue anglaise, Paris, Éditions du Cercle de la Librairie, (coll. «Bibliothèques »). 
DIAZ, C. M., (1982), Conocimiento y realidad en la mandukya upanishad y gaudapada, Madrid, Universidad de Madrid, dep. de metafisica.

DRASKAU, J.-K., PICHT, H. , (eds), (1994), International Conference on Terminology science and Terminology Planning, Riga, 17-19 August, International IITF-Workshop, Theoretical issues of terminology science, Riga 19-21 August 1992, Vienna, International Network for Terminology (IITF series ; 4).

DUBUC, R., (1992), Manuel pratique de terminologie, Montréal, Linguatech, Paris, Conseil International de la langue française.

ECO, U., (1999), Kant et l'ornithorinque, Paris, Bernard Grasset.

ESTEBAN, Miguel A., (1996), « Los lenguajes documentales ante el paso de la organización de la realidad y el saber de la organización de conocimiento ", Scrire, representación y Organización del Conocimiento, vol. 1, $\mathrm{n}^{\circ}$ 2, julio-diciembre, pp. 43-71.

FELBER, H., (1994), « East-West cooperation in international terminology work in the 1930s : Drezen and Wüster » in DRASKAU and PICHT, pp. 40-48.

FRAWLEY, W., (1985), « Intertextuality and the dictionary : toward a deconstructionist account of lexicography », Dictionaries, journal of the Dictionary society of North America, $\mathrm{n}^{\circ} 7, \mathrm{pp} .1-20$.

FREUD, S., (1968), Métapsychologie, Paris, Gallimard.

GALINSKI, C., K.-D. SCHMITZ (eds), (1996), TKE ‘96, Terminology and Knowledge Engineering, Frankfurt /Main, Indeks Verlag.

GAUDIN, F., (1993), « Socioterminologie : propos et propositions épistémologiques », Le Langage et l’homme, vol. XXVIII, ${ }^{\circ} 4$, décembre Numéro spécial « Socioterminologie », pp. 247-258.

GENTILHOMME, Y., (1994), «L'éclatement du signifié dans les discours scientifiques », Cahiers de lexicologie, $\mathrm{n}^{\circ}$ 64, 1, Paris, Didier Erudition, pp. 5-35.

GIFFARD, A., (1997), « Petite introduction à l'hypertexte », in Banques de données et hypertextes pour l'étude du roman, N. FERRARD (ed.), Paris, Presses Universitaires de France, pp. 99-117.

GOUADEC, D., (1992), « Première séance plénière : Terminologie et terminographie le point de la situation », inD. GOUADEC (ed.), Terminologie et terminotique, Actes de la première université d'automne en terminologie, Rennes 2, 21-26 septembre 1992, Paris, Maison du Dictionnaire, pp. 47-54.

GUESPIN, L., (1990), « Socioterminology facing problems in standardization », in TKE ‘90, pp. 642-648.

HALL, W., DAVIS, H., and HUTCHINGS, G., (1996), Rethinking Hypermedia ; The Microcosm Approach, Boston/Dordrecht, London, Kluwer Academic Publishers.

HANDKE, J., (1995), « Mental and machine-readable lexicons : What can machines learn from the world ", in REN, DIRVEN and J. VANPARYS (eds), Current Approaches to the lexicon; A Selection of Papers presented at the 18th LAUDSYmposium, Duisburg, March 1993, Frankfurt am Main, Peter Lang, pp. 273-299 (coll. Duisburg Papers on Research in Language, band 24).

HANDWERKER, B., (1989), « Savoir lexical, savoir expert : problème de représentation », DRLAV, $\mathrm{n}$ ${ }^{\circ} 40$, pp. 63-87.

LERAT, P., (1997), « Le contenu terminologique au péril de l'interdisciplinarité. Le cas de connaissances ", in C. de SAETZEN(ed), Terminologies et interdisciplinarité, Louvain-la-Neuve, Peeters, pp. 5-13.

LEROT, J., (1997), « Qu'est-ce que le lexique conceptuel » in Terminologie et interdisciplinarité, C. de SAETZEN (ed.), Louvain-la-Neuve, Peeters, pp. 15-35. 
LEVY, P., (1995), l'Intelligence collective, Pour une anthropologie du cyberspace, Paris, Éditions La Découverte (coll. « Sciences et société »).

LEVI-STRAUSS, C., (1987), Race et histoire (1952), suivi de l'œuvre de Claude Lévi-Strauss par J. POUILLON, Paris, Denoël.

LOFFLER-LAURIAN, A.-M., (1994), « Réflexions sur la métaphore dans les discours scientifiques de vulgarisation ", Langue française, $n^{\circ} 1, \mathrm{pp} .72-79$.

MAIGNIEN, Y., (1995), « La Bibliothèque Virtuelle ou de l'Ars memoria Xanadu », Bulletin des bibliothèques de France, t. 40, $\mathrm{n}^{\circ}$ 2, pp. 8-17.

MARIN, L., (1991), « Ruptures énonciatives dans la représentation picturale », in H. PARRET (ed.), le Sens et ses hétérogénéités, Paris, Éditions du CNRS, pp. 219-231.

MAYER, R. and HENNE, K.-H., (1990), « How to bring hypertext to termbanks »,in Terminology and Knowledge Engineering, vol. 2.

McCARTHY, J., (1985), « Epistemological problems of artificial intelligence », in R.-J. BRACHMAN and H.-J. LEVESQUE (eds), Readings in Knowledge representation, San Mateo (Calif.), Morgan Kaufman publishers, pp. 23-30.

MEAGHER, M. E. and CASTANOS, F., (1998), « Perception of American culture : The impact of an electronically-mediated cultural exchange program on Mexican high school students ", in S. C. HERRING (ed.), Computer mediated Communication ; Linguistic, Social and Cross-cultural Perspectives, Amsterdam/Philadelphia, Johns Benjamins Company, pp. 187-203.

MESCHONNIC, H.,(1996), « L'encyclopédie sortant de son mot pour se voir »in SCHAER, pp. 19-25. MERTEN, P., (1992), « Apport des relations notionnelles à la description terminologique », communication présentée à TAMA ‘92 (Avignon).

MERTEN, P., MERTENS, J. et Van CAMPENHOUDT, M., (1992), « Microglossaire, réseau notionnel et gestion informatique ; une expérience de recherche en Communauté française de Belgique », inD. GOUADEC (ed.), Terminologie \& Terminotique; outils, modèles et méthodes; Actes de la Première Université d'Automne en terminologie, Rennes 2-21 au 26 Septembre, Paris, La Maison du Dictionnaire, pp. 277-293.

MORGENROTH, K., (1994), le Terme technique, Approches théoriques, études statistiques appliquées à la langue de spécialité économique du français et de l'allemand, Tübingen, Max Niemeyer Verlag, VII, (coll. Beihefte zur zeitschrift für romanische Philologie, Band 250).

MORTUREUX, M.-F., (1995), « Le vocabulaire scientifique et technique »,in J.-C. BEACCO et S. MOIRAND, (eds), les Enjeux des discours spécialisés, Les Carnets du Cediscor, Paris, Presse de la Nouvelle Sorbonne, pp. 13-26.

NEUSNER, J., (1997), « Paradigmatic versus historical thinking : the case of Rabbinic Judaism », History and Theory, Studies in the Philosophy of History, vol. $36 \mathrm{n}^{\circ}$ 3, Wesleyan University, pp.

353-377.

OESER, E., (1994), « Terminology and philosophy of science », in DRASKAU and PICHT, pp. 24-34.

OTMAN, G., (1994), « Pourquoi parler de connaisssances terminologiques et de bases de connaissances terminologiques », La Banque des mots, nº́cial 6, pp. 5-27.

OTMAN, G., (1996), Les représentations sémantiques en terminologie, Paris, Barcelone, Milan, Masson, VII.

PARRET, H., (1991), «Introduction », inH. PARRET (ed.), Le sens et ses hétérogénéités, Paris, Éditions du CNRS, pp. 11-25. 
PASTOR SANCHEZ, J. A., and PEREZ T. S., (1996), « Un interfaz hipertexto para un sistema de gestión de Tesaurus », Scrire, Representación y Organización del conocimiento, Vol. 2 nº 1 , enero-junio, Universidad de Zaragoza, pp. 51-62.

PIOTROWSKI, D., (1996), « Opérateurs hypertextuels et formes lexicographiques » in D. PIOTROWSKI (ed.), Lexicographie et informatique; autour de l'informatisation du Trésor de la langue française, Actes du colloque International de Nancy (29-30-31 mai 1996), Paris, Didier Erudition, pp. 319-336.

PITZ, K., (1997), « Complexity : a paradigm for 'one culture'? », Forum Italicum, vol. $31 \mathrm{n}^{\circ}$ 2, pp. 423-437.

RAJAGOPALAN, R., (1996), « A interdisciplinaridade : um imperativo desde sempre », Boletim da Associação Brasileira de Lingüistica, nº 19, Maceió, Brazil, Impresa Universitária, UFAL, pp. 93-103.

RASTIER, F., (1996), « Problématiques du signe et du texte », Intellectica, n², pp. 11-52.

RASTIER, F., (1995), « Le terme : entre ontologie et linguistique », La Banque des mots, nº spécial 7 , pp. 35-65.

RASTIER, F., (1991), « l'Analyse linguistique du texte expert », in Actes du colloque Génie linguistique, Versailles, janvier, document tapuscrit, [s.p.].

ROBERTSON, R., (1996), « Globality and transdisciplinarity », [compte rendu de « Cultural identity and Global Process, J. FRIEDMAN, London, Sage publications » 1994], in Theory, Culture and Society, «Exploration in Critical social Science », vol. 13, $\mathrm{n}^{\circ} 4$, Sage, London, New Delhi, Thousand books, pp. 127-132.

ROJO, A., (1995), « La invención de maquinas simuladoras de los procesos heurísticos de pensamiento ", Anthropos, revista de documentación cientifica de la cultura, n¹64, enero, Barcelona, pp. 33-40.

ROJO, A., (1992), Carnegie Mellon University : culturas de invención ingenieria informatica ; culturas de invencion social, doctorate thesis, dept. of anthropology, University of Barcelona.

ROJO, A., (1995), « Hypermedia decision net un nuevo paradigma docente profesionaltelematico », Barcelona, [Universitat de Barcelona], [circa].

ROJO, A., (1996), and CABRÉ, T., « Specialized knowledge representation : towards a new hypertextual /multimedia proposal »,in TKE ‘96, pp. 414-423.

SAGER, J. C., (1990), A practical Course in Terminology Processing, Amsterdam/ Philadelphia, John Benjamins Publishing Company, XI.

SCHAER, R. (ed.), (1996), Tous les savoirs du monde. Encyclopédies et bibliothèques, de Sumer au XXIe siècle, Paris, Bibliothèque nationale de France, Flammarion.

SIMMEL, G., (1995), « Le problème du temps historique », pres. et trand. de J.-L. Evard, Revue de métaphysique et de morale, 100e année, $\mathrm{n}^{\circ}$ 3, pp. 295-309.

SLODZIAN, M., (1994), « Terminology theory and philosophy of science », in DRASKAU and PICHT, pp. 35-39.

SLODZIAN, M., (1995), « Comment revisiter la doctrine terminologique aujourd'hui », La Banque des mots, $\mathrm{n}^{\circ}$ spécial 7, pp. 11-18.

TEMMERMN, R., (1997), « The process of lexicalization. The case of the life sciences », in De SAETZEN, pp. 47-62. 
TODT, O., (1997), « Ingenieria y controversia social : hacia una nueva forma de diseño de sistemas tecnicos ", Arbor, Madrid, t. CLVII (julio), pp. 167-182.

TRICOT, A., PIERRE-DEMARCY, C. et EL BOUSSARGHINI, R., (1998), « Un panorama des recherches sur l'activité mentale de l'utilisateur d'un hypermédia », in E. BRUILLARD, B. de LA PASSARDIERE et G.-L. BARON, (eds), Le livre électronique, Sciences et techniques éducatives, 5, 4, pp. 371-400.

WUSTER, E., (1996), Terminologia, selecció de textos d'E. Wüster, M. T. CABRE I CASTELLVI (ed.), Barcelona, Servei de llengua catalana.

WUNENBURGER, J.-J., (1990), la Raison contradictoire, science et philosophie modernes : la pensée du complexe, Paris, Albin Michel.

\section{Numéros Spéciaux}

Le Langage et l'homme, vol. XXVIII, « Socioterminologie ».

Alfa, $n^{\circ} 7 / 8$.

La Banque des mots, $\mathrm{n}^{\circ}$ 6, 1994.

La Banque des mots, $\mathrm{n}^{\circ}$ 7, 1995.

\section{NOTES}

1. Voire employé sous la forme terminotics. Cf. International Conference on Computational Linguistics (6: 1977) Coling, Ottawa, Canada

2. $1997: 128$.

3. Créé par Ted Nelson.

4. Voir Winograd, cité par Cook, $1994: 61$.

5. Traduction de « were relying on a comitee of specialists [...] to fix its terminology whereas on the other side, the socioterminological one, we have to deal with various uses of speech » (L. Guespin, 1990: 645).

6. Voir A. Assal, 1995.

7. Jean-Jacques Wunenburger affirme : «La raison humaine parvient [...] à canaliser le multiple par des systèmes classificatoires, par des langages et des codes, à stabiliser le mouvant par une logique identitaire qui amarre le flux et reflux des choses à des principes simples et des substances en nombre limité.» (1990: 10). Voir aussi dans le même ouvrage, p. 138: «La relativisation de la pensée identitaire passe en ce sens par une pluralisation des procédures de représentation et d'énonciation, reposant sur un éclatement du modèle logico-grammatical qui la gouverne. »

8. Traduction libre de "The scientific study of specialized vocabulary is confounded with the pragmatic activity of standardization. This urge for standardization is a by product on objectivism, which is deeply rooted in the Western philosophical and cultural tradition » (Rita Temmerman, 1997: 49).

9. Rastier, 1991 : f. 2.

10. Voir aussi Otman, 1996: 161-162, pour les liens multidimensionnels et les relations nonhiérarchiques.

11. Les dictionnaires bilingues tentent même de réduire le vocabulaire définitionnel au minimum afin que l'apprenant puisse comprendre aisément.

12. Traduction libre de: «A scientific lexicon [Kuhn] is constitutive of a phenomenal world in the sense that it creates a taxonomy of kinds corresponding to the concepts available to the speech community living in the world. » (Ahmad, 1996 : 9) 
13. $1991: 219$.

14. Marie-France Mortureux donne l'exemple de la respiration en physique qui est définie comme un phénomène d'oxydation et celle de la biologie comme celui d'un échange gazeux (1995: 21).

15. Sur une approche interdisciplinaire de la culture, voir Pilz, $1997: 423$.

16. Voir Otman, 1996: 161-162.

17. 1997: 23

18. Mary Elaine Meagher et Fernando Castanos (1998: 199) affirment: «If we define culture in an anthropological and social sense as including attitudes, values, thought patterns, frames of reference and daily activities we can say that electronically-mediated exchange programs increase contact with the culture of L2. "

19. Voir Rojo, 1992

20. Neusner 1997 : 371. Voir aussi Otman 1996: 92 parlant du concept de temps mythique, in J. De Kleer et J. S. Brown (eds), 1984.

21. Freud (1968: 12) affirmait déjà que les concepts fondamentaux de la physique étaient en constante modification. Jean-Pierre Casseyre (1994 : 172) donne un exemple de ce problème dans l'histoire de la classification des bibliothèques : « Les notions utilisées dans certaines des classes de la CDU ont été rapidement dépassées par l'évolution des idées scientifiques, et la classification n'a été capable d'intégrer les notions nouvelles (en physique par exemple) que de manière purement artificielle. »

22. Traduction libre de: «The interdependency of conceptual change and the modification of hypothesen theories, etc. during the research process is accompanied by another phenomenon typical for the social sciences and the humanities: unlike natural sciences such as physics, etc. these disciplines do not have any predominant paradigm (in the sense of T. S. Kuhn) but rather a plurality of theories, hypotheses, schools of thought etc. concerning the same 'objects'. This means that several differing concepts are formed about the same object according to the framework of the respective theory. "

23. On notera avec Herman Parret (1991 : 14), que la phénoménologie a pu s'allier si facilement avec l'herméneutique : « le texte à déchiffrer, les discours à "traduire”, les paroles à interpréter, les cultures à comprendre, voici encore la dominance du sens présupposée par la technique herméneutique de transposition. " Cette position semble éclairer la démarche herméneutique et paradigmatique de l'exploration hypertextuelle.

24. Youssef Assy, 1995: 547. Sur le sens de paradigme chez les pionniers de la théorie linguistique, voir l'auteur, pp. 541-547.

25. Voir Pastor Sanchez and Perez, 1996 : 51, et Condamines, 1994 : 37-38.

26. Sur le lien hypertexte, voir Hall, Davis and Hutchings, 1996.

27. Traduction libre de: «A dictionary should be an encyclopedic database of the words of a language or languages coupled with an open-ended set of deductive access method and multimedia display methods » (1996: 12).

28. Otman écrit : « Il est admis qu'un terme scientifique ne peut fonctionner qu'à l'intérieur d'un système notionnel et qu'il tire son sens des liens [...] qu'il entretient avec l'ensemble des éléments de ce système.»(1996:126). L'organisation hiérarchique et la recherche terminologique demanderait-elle, au contraire de l'organisation réticulaire, une connaissance élémentaire dans le domaine (Voir aussi R. Dubuc, 1992 : 38).

29. 1995 : 202. Voir aussi K. Rajagopalan, 1996. Rajagoplan affirme que les diverses vérités locales concernant un même objet sont complémentaires. 


\section{RÉSUMÉS}

La représentation linéaire de la connaissance ne peut rendre compte de la nature polyèdre du terme. Le néologisme hyperterminotique désigne les situations ou processus d'organisation et de gestion de termes et de terminologies par le biais d'outils hypertextuels à l'intérieur des réseaux. L'hyperterminotique débouche sur une définition éthique du concept de paradigme.

A linear knowledge representation cannot assume the polyhedral nature of terms. What we call hyperterminotics includes situations or processes of term and terminology organization and management through hypertextual tools within networks. Hyperterminotics brings forth an ethics-based definition of paradigm.

\section{AUTEUR}

\section{JEAN-NICOLAS DE SURMONT}

B.P. 2065

54100 - Nancy

bouddha@mailexcite.com 